\title{
La sinagoga del barrio de Caleros
}

Jean PASSINI *

CNRS

Francisco Cantera Burgos ${ }^{1}$ señala en 1973, basándose en un documento del monasterio de Santo Domingo el Real fechado en 1355, la existencia de una sinagoga en el toledano barrio de Caleros (Figuras 1 y 2). Julio Porres ${ }^{2}$ consideraba a este respecto que «como la sinagoga citada en la calle o en el barrio de Caleros, pero no en la plaza, debió tener su entrada por la del Aljibillo», por un corto adarve. El análisis y confrontación de documentos de los siglos XIVXV del monasterio de Santo Domingo el Real y del hospital de la Misericordia nos permite fundamentar y precisar la localización de la sinagoga en el llamado «barrio de Caleros» (figura 2). Tal es el objeto de este artículo.

\section{Documentación}

\section{A propósito de la sinagoga de Caleros}

Nos referiremos en primer lugar a los documentos que mencionan Cantera Burgos y Porres. El primer documento que se refiere a la sinagoga, fechado en octubre de 1355, dice que doña Inés, hija de Alfonso Yañes y viuda de Juan Alfonso Carrillo, vende a su nuera Teresa Alfón una casa en Toledo, «en la collaçión de Sant Salvador, en el barrio de los

\footnotetext{
*jpassini@free.fr

1 F. Cantera Burgos, Sinagogas de Toledo, Segovia y Córdoba (Madrid 1973).

2 J. Porres Martín-Cleto, «La judería de Toledo después de la expulsión», en La expulsión de los judíos de España [= II Curso de Cultura Hispano-Judía y Sefardí (Toledo, 16-19 sept. 1992)] (Toledo 1993), págs. 26-45.
} 
Caleros, tiene con una parte con la xinoga de los judíos, y de la otra parte con casa que fueron de Alfonso Gómez, criado de Juan Pérez Suárez de Meneses» ${ }^{3}$. En 1402, Mencía García, monja de Santo Domingo el Real, da a censo al pellejero Juan González de Bonilla una casa «... con cuatro tiendas que son ensomo de ellas en la colación de Santo Tomé en el barrio de los Caleros, que linda con la xinoga de barrio de Caleros» ${ }^{4}$; a ella se la había donado en 1396 Tel Fernández de Toledo ${ }^{5}$. En noviembre de 1411, Juan González de Bonilla y su mujer reconocen «que tienen a censo de soror Mencía González, monja de Santo Domingo el Real unas casas con cuatro tiendas encima... que lindan con la sinagoga...» ${ }^{6}$ por 700 maravedís; pero como la casa estaba en malas condiciones «se las devuelven a la monja». Siete años después, en mayo de 1418, la priora Teresa de Ayala y la comunidad aprueban una permuta con Pedro Díaz Alhanet «de unas casas y tres tiendas que están en el barrio de Caleros, que lindan con la sinagoga y que son de soror Mencía García de Toledo y de su hija soror Urraca Téllez...» 7 . En octubre del mismo año, María Álvarez y Teresa de Ayala firman una carta de censo de 260 maravedís al zapatero Fernando Sánchez Bazuelo y a su mujer por una tienda «con sus entradas y salidas» al barrio de Caleros ${ }^{8}$. Tras la muerte de Urraca y de Mencía Téllez, en 1418, el monasterio de Santo Domingo el Real dio las casas de Mencía García, que lindaban con la «xinoga de barrio de

3 AHN, Clero, pergs, carp. 3072, n 3 (transcripción A. Yuste); y P. León Tello, Judios de Toledo (Madrid 1979), t. II, doc. 511.

4 León Tello, Judios de Toledo, pág. 684; Cantera Burgos, Sinagogas, págs. 54-55.

5 Becerro de Santo Domingo el Real, 1396: «... donacion que hizo... Tel Fernandez de Toledo... y de unas casas que el tenia en Toledo en barrio de Caleros... con las tiendas que en ellas son y salen las puertas de ellas a la calle...» (transcripción A. Yuste). Tres años después, estos bienes se donan a su hija natural, Urraca Téllez (Becerro de Santo Domingo el Real, 1399).

6 AHN, Clero, carp. $3080 n^{\circ} 8$.

7 AHN, Clero, carp. 3082 n 5: «...y tiene a tributo de 700 mrs. Pero Díaz y se ha convenido con las propietarias que dejase estas casas, dandole a cambio otras en la colación de San Andrés y por el trueque 2000 maravedis».

8 AHN, Clero, carp. $3082 \mathrm{n}^{\circ} 7$. 
Caleros» ${ }^{9}$, a Teresa Téllez, que a partir de $1423{ }^{10}$ da a censo por separado casa y tiendas (figura 3 ).

Como vemos, la sinagoga de Caleros aparece en contratos de venta o de alquiler desde 1355 a 1418 como linde de una casa y de las tiendas anejas a esta. Solo conocemos uno de los inmuebles aledaños a la sinagoga: se trata de una casa con varias tiendas encima, que eran cuatro a fines del siglo XIV y solo tres a comienzos del XV, que Teresa Téllez alquila por separado de la casa a partir de 1423. Los documentos citados no nos permiten localizar en el barrio ni la sinagoga ni la casa; sin embargo, contratos de alquiler posteriores ${ }^{11}$ nos posibilitan situarlas con exactitud.

\section{La casa y las tiendas próximas a la sinagoga de Caleros}

Un contrato de alquiler fechado en 1455 dice que la casa de Teresa Téllez (Santo Domingo [= SD]-74) está entre las tiendas y una casa tributaria del hospital de la Misericordia a la que nos referiremos más adelante. En 1460, la casa se sitúa entre las tiendas y la casa de Johan de Écija, que estaba más abajo (apéndice documental $n^{\circ} 1$ ). Una de las cámaras da «por la calle de arriba que sale al cobertizo del arçediano de Niebla». También leemos que una de las tres tiendas (SD-77), «... linde de la suso dicha (SD-76) e de la otra parte de arriba con casas que fueron del arçediano de Niebla, la cual viene sobre las casas del dicho Diego Lopes de Orgas (SD-74) e alinda con la dicha calle...». Más adelante, en $1501^{12}$, la casa se describe en el «barrio de Caleros», entre «de una parte con casas que fueron de Johan de Écija fustanero y son agora de Alvar de San Martín tributario del Hospital de la Misericordia..., e de la otra parte con casas e tiendas del dicho monasterio que son en la calzada a barrio de Caleros

9 LeÓn Tello, Judios de Toledo, doc. 739.

10 AHN, Clero, pergs. carp. 3082, n 5 y 7, citado por J. L. Barrios Soto, Santo Domingo el Real y Toledo a fines de la Edad Media (1364-1507) (Toledo 1997).

11 La casa de Teresa Téllez sería alquilada por Alfonso Cordero en 1455, Diego López, tundidor, en 1459, el zapatero Diego López de Orgaz en 1460, el tripero Juan Fuente en 1501, Juan Alonso de Toledo en 1517.

12 ASDR [= Archivo de Santo Domingo el Real], «Asiento de todas las posesiones del monasterio de Santo Domingo el Real, comenzado en 1507», fols. CCCCLXIIIr y CCCCLXIIIIr (CD-Rom), pág. 347, fol. 267. 
e algunas de ellas vienen ençima destas dichas casas [SD-74] e la calle real...». Una de las tiendas «que están en la calzada en la calle que va a las casas del Conde de Cifuentes que fueron del arcediano de Niebla» está «al esquina de la dicha calzada que hace la calle que va hacia iglesia de San Salvador». Linda con una tienda del monasterio de Santo Domingo el Real y «viene encima de la casa... de Johan de Fuentes jubetero».

\section{La casa tributaria del hospital de la Misericordia}

Esta casa ${ }^{13}$ (HM-90a) fue comprada en 1448 al cerrajero Gonzalo López por 25000 maravedís. Lindaba entonces «... de una parte una casa de la mujer de Alfonso Gómez jurado, de otra casa que fueron de Alfonso González Cordero [SD-74] y a la espalda con casa del arcediano de Niebla y de la otra parte con calle publica real...». En 1459, el ocupante de esta casa, que limita «por una parte casa de Mayor Gómez, mujer de Ferrand Alfonso de Sevilla que Dios aya y de la otra parte casas que fueron del arcediano de Niebla y de la otra parte casa de Diego Lopez tundidor [SD-74]...», confirma el tributo al hospital. Treinta años después, el documento que ratifica la venta realizada en 1448 precisa los linderos, que eran entonces la casa de Diego López de Orgaz (SD-74) por un lado, por otro la casa de Mayor Gómez, mujer de Fernando Alfonso de Sevilla, y finalmente, la plaza a la que daba «la puerta de las casas de los Reyes...». En 1517, un documento en el que el doctor Francisco del Castillo reconoce un tributo de 900 maravedís sobre una casa comprada a Álvaro de San Martín dice que esta casa se ubica entre las del conde de Cifuentes y una que ha alquilado Juan Alonso de Toledo (SD-74).

\section{INTERPRETACIÓN}

El análisis de los documentos citados nos lleva a formular las observaciones siguientes:

Primero, la localización de la casa y de las tres tiendas de Teresa Téllez. La casa y las tres tiendas se describen en la segunda mitad del

13 ADPT [= Archivo de la Diputación Provincial de Toledo], Hospital de la Misericordia, Leg. $5, \mathrm{n}^{\circ} 8$.

Sefarad, vol. 66: 1, ENERO-JUNIO 2006, PÁGs. 55-68. ISSN 0037-0894 
siglo XIV en «la colación de San Salvador» y en el barrio de los Caleros; los documentos posteriores dicen que esos inmuebles están en la parroquia de Santo Tomé y en el mismo barrio. Esto no es en modo alguno contradictorio, ya que las dos parroquias se extendían sobre territorios limítrofes; significa más bien que casa y tiendas estaban en el barrio de los Caleros, en el límite entre las dos parroquias ${ }^{14}$.

En aquellos años formaban una unidad situada entre una casa que fue de Juan Pérez Suárez de Meneses ${ }^{15}$ y una sinagoga. Hacia 1425, casa y tiendas, propiedad de Teresa Téllez y afectas a un tributo a favor del monasterio de Santo Domingo el Real, se alquilan por separado. La casa tiene una fachada a la calle del Salvador y se describe entre las tiendas de Teresa Téllez (SD-75, SD-76, SD-77), y una casa que tributa al hospital de la Misericordia (HM-90a). Está adosada, así como una de las tiendas, a la casa del arcediano de Niebla. Una de las cámaras da en 1460 «a la calle de arriba que sale al cobertizo del arçediano de Niebla». En 1501, la casa se sitúa entre la esquina que forman las actuales calles de Alfonso XII (antigua de Caleros) y de Rojas, y la plaza del conde de Cifuentes.

El análisis conjunto de la descripción y mediciones del Libro de las Medidas de Santo Domingo el Real (Documento $\mathrm{n}^{\circ} 1$ del apéndice), que se tomaron en 1460, y de la construcción actual, nos permite identificar la casa de Teresa Téllez con el $n^{\circ} 6$ de la calle de Rojas ${ }^{16}$, en la esquina de la actual plaza de Marrón, junto a la calle de Alfonso XII. Nos ha facilitado también la restitución de la casa de Teresa Téllez y de las tres tiendas (figura 3).

Segunda observación, la ubicación de la sinagoga de Caleros. La sinagoga estaba adosada a la casa de Teresa Téllez (SD-74) y a una de las tiendas (SD-77). Sabemos que la casa SD-74 se levantaba en la esquina de las calles de Caleros y San Salvador, y conocemos también cuál fue el devenir de la casa HM-90a «que fueron de Alfonso Gomez» (Anejo 2);

14 La parroquia de San Salvador perdió su autonomía tras la reforma municipal de 1422, tratada por J.-P. MolÉnAt en «L'oligarchie municipale de Tolède au XV siècle», en Tolède et l'expansion urbaine en Espagne (1450-1650) (Madrid 1988), págs. 159-177. J. Porres, Historia de las calles de Toledo, tomo III ( $3^{a}$ ed. Toledo 1988), págs. 1486-1487, señala que «en 1401, se adjudica el barrio de Caleros a la colación de Santo Tomé».

15 Ver nota 5.

16 Damos las gracias al propietario de esta casa, que nos autorizó a visitarla y tomar medidas. 
así, aparece claramente que la sinagoga estaba pegada a la tienda SD-77 y a espaldas de la casa de Teresa Téllez (SD-74) y de la del hospital de la Misericordia (HM-90a). La sinagoga fue incluida más adelante en los bienes del arcipreste de Niebla, para ser reemplazada a fines del siglo XV por una plaza llamada «de la puerta de los Reyes».

\section{DisCUSIÓN}

La casa de Teresa Téllez, a la que estaba pegada la sinagoga de Caleros, abría a la estrecha calle que desciende hacia la iglesia del Salvador, y las tiendas a la calle principal del barrio de los Caleros, que se llamaba calle de Caleros (actual calle de Alfonso XII). En esta última había un cobertizo, que se llamó sucesivamente del arcediano de Niebla ${ }^{17} \mathrm{y}$ del conde de Cifuentes ${ }^{18}$. Este cobertizo existía aún en el siglo XIX y figura en el plano de Coello ${ }^{19}$.

Es probable que, al igual que las demás sinagogas de Toledo cuyo recuerdo transmitió el poema de Albené ${ }^{20}$, la de Caleros padeciera los efectos del movimiento antijudío de 1391. Abandonada en este tiempo o poco después, no se la vuelve a mencionar a partir de 1418. En 1448 reaparece, ahora como casa propiedad del arcediano de Niebla, que desde $1434^{21}$ fue adquiriendo casas de este barrio para su patrimonio, una cuarta parte del cual compró en 1460 Juan de Silva, conde de Cifuentes.

El inventario de los bienes del arcediano ${ }^{22}$ nos permite comprobar que a lo largo de la alta calle «de Caleros», las casas alternaban con

17 AHN, Nobleza, leg. 248, n 1 . El conde de Cifuentes compró en 1460 la cuarta parte de los bienes del arcediano de Niebla a sus herederos.

18 Sebastián de Horozco, Relaciones históricas toledanas, pról. y transcr. de J. Weiner (Toledo 1981). El cobertizo del conde de Cifuentes lo cita Orozco cuando relata el trayecto del cortejo de los reyes don Felipe y doña Isabel: «Luego su majestad fue por el cobertizo del conde de Cifuentes y por la calle del canonigo Mariana. Y salio a la Plazuela de Sant Salvador».

19 F. Quirós LinARES, Las ciudades españolas en el siglo XIX, Vistas de ciudades españolas de Alfred Guesdon, Planos de Francisco Coello (Valladolid 1991), pág. 261, y Toledo, Plano de escala 1/5.000 (Madrid 1858).

20 Cantera Burgos, Sinagogas, op. cit.

21 AHN, Clero, carp. 3086, fol. 13.

22 AHN, Clero, leg. 7336. 
estrechas travesías que desembocaban en la calle de la Trinidad. Una de ellas, conocida como callejón de Marrón a partir de $1858{ }^{23}$, todavía subsiste; el trazado de las otras se nota aún en el parcelario actual. La casa principal del conde de Cifuentes, llamada «de los sennores Reyes» en la segunda mitad del siglo XV, en la que los reyes se solían alojar en la segunda mitad de ese siglo y aún durante el siglo siguiente, ocupaba una gran manzana a la altura del cobertizo de Caleros. Al otro lado del cobertizo se situaba, en la esquina de la calle de Caleros con el cobertizo de San Pedro Mártir, la casa «accesoria», resultante de la fusión de tres pequeñas casas ${ }^{24}$. Delante de la puerta de la casa principal del conde de Cifuentes se abriría una plaza en el último cuarto del siglo XV ${ }^{25}$, sobre el solar de la sinagoga (Figura 5), una vez que esta desapareció por ruina o por derribo intencionado.

Este intento de comprensión de la evolución \{ sinagoga $\rightarrow$ casa del arcediano de Niebla $\rightarrow$ plaza de la puerta de la casa del rey $\}$ nos lleva a formular como hipótesis que la sinagoga de Caleros abría no a la calle del mismo nombre, sino al callejón de Marrón (Figura 4). Invalida la propuesta de J. Porres, que emplazaba la sinagoga en el callejón de Aljibillos.

\section{CONCLUSIÓN}

El estudio presentado, al mismo tiempo sitúa con precisión la sinagoga en la calle principal del barrio de Caleros, no muy lejos del cobertizo de Caleros, y enriquece nuestro conocimiento del barrio. La calle principal de este parece haber sido una vía de intensa actividad comercial en la segunda mitad del siglo XIV. Al nivel de nuestras investigaciones, consideramos que el cobertizo de Caleros marcaba la entrada del barrio judío del mismo nombre, lo que da a parecer una analogía entre la sinagoga de Caleros y la del Sofer ${ }^{26}$, ambas están ubicadas no lejos de la entrada a la

23 J. Porres Martín-Cleto, Historia de las calles de Toledo (4 ${ }^{\mathrm{a}}$ ed., Toledo, 2002) tomo II («Travesía (hoy plaza) de Marrón»), págs. 685-687.

24 Juan de Rojas, secretario del conde de Cifuentes, vivía en esta casa en 1475. ADPT, libro 849.

25 Esta plaza se menciona por primera vez en un documento de 1489. Llevará hasta el siglo XIX el nombre de plaza o plazuela de Cifuentes.

26 J. PAssinI, «La sinagoga del Sofer en Toledo», Sefarad 64 (2004), págs. 141-157. 
calle principal de un barrio judío, acceso marcado bien por un cobertizo, bien por una puerta. Esta modesta aportación a nuestro conocimiento no debe enmascarar la ignorancia en la que aún nos movemos acerca de la Judería de Toledo y la necesidad de velar por que cualquier intervención urbanística en el barrio actual sea cuidadosamente meditada.

\section{APÉNDICE DOCUMENTAL}

\section{1}

Medidas de casas de Santo Domingo el Real de Toledo, 1460

\section{ANH Clero, libro $15118^{27}$}

[SD-74] Yten otras casas en la dicha collaçion de Santo Thome a barrio de Caleros, las quales solian ser de Alfon Gonçales Cordero e tyenelas agora a çenso infiteosyn Diego Lopes de Orgas çapatero, por nueveçientos mrs. cada anno, que han por linderos de la una parte casas del dicho monesterio de partes de arriba e de partes de ayuso casas de Johan de Eçija fustanero, en las quales dichas casas ay una casa puerta con un establo que ay en luengo en todo siete varas e tres quartas e en ancho dos varas e dos terçias. Yten una cosina a par del dicho portal como entran a la mano derecha e un portal adelante della que ay en luengo en todo honse varas e de ancho tres varas e todo esto entrada e establo e cosina e portal esta todo en su altura fasta el çielo e fasta los abismos. Yten ay mas un palaçio como entran en las dichas casas a la mano ysquierda e mas adelante $/ \mathrm{f}^{\circ} 23$ $\mathrm{v} \%$ del en la dicha hasera una bodega todo de largura de quinse varas e de ancho quatro varas e la camara de ençima deste dicho palaçio e bodega no es destas casas salvo de otras casas tributarias al dicho monesterio que se mandan por la calle de arriba que sale al cobertiso del arçediano de Niebla. Yten delante deste palaçio esta un portal que es cubierto ençima de una camara que es de las dichas casas lo alto e baxo que ay en luengo honse varas e en ancho dos varas e terçia que sale al ayre del corral. Yten ay más en las dichas casas un palaçio frontero como entran en ellas que ay de luengo siete varas e media e de ancho tres varas e ençima deste dicho palaçio esta una camara de la dicha anchura e largura armada a un agua. Yten delante deste dicho palaçio esta un portal que sale al ayre de la casa do está el escalera e ençima otro portal delante la camara que es de la largura del dicho palaçio. Yten en el patyn destas dichas casas ay en luengo siete varas

27 Transcripción de J.-P. Molénat. 
e media e en ancho tres varas e de partes de fuera destas casas fasya el esquina de arriba de la calle esta una casilla que es destas dichas casas que ay en luengo della quatro varas e en ancho tres varas e siete ochavas e la camara de ençima della no es de las dichas casas salvo de las casas que tiene Johan Lopes cardador e por dentro de esta casilla se manda la camara que viene sobre la entrada e el establo de las dichas casas del dicho Diego Lopes. A lo qual estovo presente el dicho Diego Lopes de Orgas.

[SD-75] Yten una casa a la dicha collaçion de Santo Thome al dicho barrio de Caleros al esquina de la pared, la qual viene ençima de la casilla e del palaçio de las dichas casas del dicho Diego Lopes de Orgas e alinda con las dichas casas del dicho Diego Lopes e de la otra parte con casas del dicho monesterio e esta casa suben en alto a ella e tiene un poyo delante quanto es su pertenençia de una vara en ancho, la qual casa tiene perpetua por su vida e de su muger e de un fijo $/ \mathrm{f}^{\circ} 24 \mathrm{r}^{\circ} /$ Johan Lopes cortidor por dosientos e sesenta mrs. cada anno, en la qual casa ay de luengo seys varas e una ochava e de ancho quatro varas e tyene ençima una camara desta mesma longura e sale sobre la puerta sobre canes a la calle e con la gordura de la pared una vara mas que lo de baxo de ancho e tiene su altura fasta el çielo.

[SD-76] Yten otra casa en linde de la suso dicha en la dicha collaçion de San Thome que es del dicho monesterio, la qual solia tener del dicho monesterio Maria Gonçales panadera e de la otra parte alinda con otra casa del dicho monesterio que tiene Davi sastre e ay en esta casa de luengo seys varas e de ancho quatro varas, la qual viene sobre el palaçio de las casas del dicho Diego Lopes de Orgas e tiene esta casa con toda su pertenençia en la calle un poyo alto de una vara en ancho. Yten tyene mas esta casa una camara ençima de la dicha largura e su salida sobre canes al corredor aque sale de ancho mas que lo de yuso dos varas porque sale un poco más sobre las casas del dicho Diego Lopes de Orgas e tiene su altura fasta el çielo.

[SD-77] Yten otras casas en linde de la suso dicha e de la otra parte de arriba con casas que fueron del arçediano de Niebla, la qual viene sobre las casas del dicho Diego Lopes de Orgas e alinda con la dicha calle, las quales tyene perpetuas don Davi sastre por su vida e de su muger e de un fijo o fija por dosientos e sesenta mrs. e un par de gallinas cada anno e ay en el luengo della seys varas e en ancho quatro varas e su pertenençia de poyo delante quanto la salida de la 
madera de los canes de la camara. Yten tyene esta casa una caámara ençima en que ay de luengo seys varas e quarta e de ancho con lo que sale sobre canes a la calle çinco varas e ochava e su altura es fasta el çielo e tiene mas esta casa una açutea que se manda por la dicha camara, la qual açutea viene sobre el palaçio pequenno de las casas del dicho Diego Lopes de Orgas e es de largo dos / $\mathrm{f}^{\circ} 24 \mathrm{v}^{\circ}$ / varas e quarta e de ancho del palaçio debaxo que es de Diego Lopes e su altura fasta el çielo. ccLx.

\section{2}

Libro memorial del testamento y bienes del canónigo Fernán Martinez de Fuensalida.

ADPT, Hospital de la Misericordia, Leg. $5, n^{\circ} 8$, fols. $55 v^{28}$

\section{Sant Salvador}

Otrossi, tiene e possee el dicho ospital un tributo de quantia de nueveçientos e treynta maravedis en cada uno año en unas casas que tiene e possee Ferrando Diaz de Uzeda que son a barrio de Caleros en que ay una casa puerta con un establo en que ay diez varas en largo e en ancho dos varas e media. Un portal entre la casa puerta e el patin en que ay dos varas en ancho e tres varas en largo. Un patin ladrillado en que ay quatro varas en quadra. Un portal en que ay siete varas en largo e en ancho tres varas. Un palaçio a la mano derecha que ha en largo seys varas e en ancho tres varas. Un portal frontero en que ay quatro varas en largo e tres varas en ancho. Un palaçio frontero que ha siete varas en largo e tres varas en ancho. En lo alto ay una camara ençima del palaçio frontero de la anchura e longura del dicho palaçio e delante de ella un corredor. Sobre el palaçio de la mano derecha e sobre la portada ay una cozina en que ay siete varas en largo e tres varas en ancho con una chimenea. Otra camara en que ha ocho varas en largo e dos varas e media en ancho. Ay una açotea que viene sobre la camara del palaçio en que ha ocho varas en largo e quatro en ancho. Otra camara sobre el portal de la mano derecha que ha seys varas en largo en el ancho a un cabo tres varas e al otro dos varas e media. Ay en el palaçio de la mano derecha un retrete. Esta possession fue dotada al dicho ospital por el dicho Alonso Sanches de Jahen, canonigo.

\footnotetext{
28 Transcripción de A.Yuste.
} 


\section{RESUMEN}

Un documento de 1355 menciona la existencia de una sinagoga en el barrio de Caleros en Toledo sin precisar el lugar. Dicha sinagoga figura en los contratos de venta de la casa vecina, propiedad de Teresa Téllez entre 1355 y 1418. El estudio de este edificio, descrito y medido en 1460, y hasta el primer cuarto del siglo XVI confrontando la descripción del mismo con la situación actual de construcción, nos conduce a situar y restituir dicha casa, y por consiguiente, la sinagoga de Caleros. El inventario de bienes del arcediano de Niebla (ca. 1434) permite precisar la situación de la sinagoga en un callejón abierto a la calle principal del barrio de Caleros. Aparte de la localización de la sinagoga, este artículo precisa los contornos del barrio de Caleros a fines del siglo XV.

Palabras clave: Sinagoga, barrio judío, Toledo, historia de los judíos en España.

\section{SUMMARY}

A document dated in 1355 mentions (though no specific location is given) the existence of a synagogue in the Caleros quarter in Toledo. The study of this building (the so-called Caleros synagogue), described and measured in 1460, and documented up to the early Sixteenth C., is done confronting its description with the present location. An inventory with the properties of the Niebla archdeacon (ca. 1434) allows us to fixing its location in a narrow street open to the main street of the Caleros quarter. An additional description of this quarter is provided.

KeYwords: Synagogue, Jewish Quarter, Toledo, History of the Jews in Spain. 


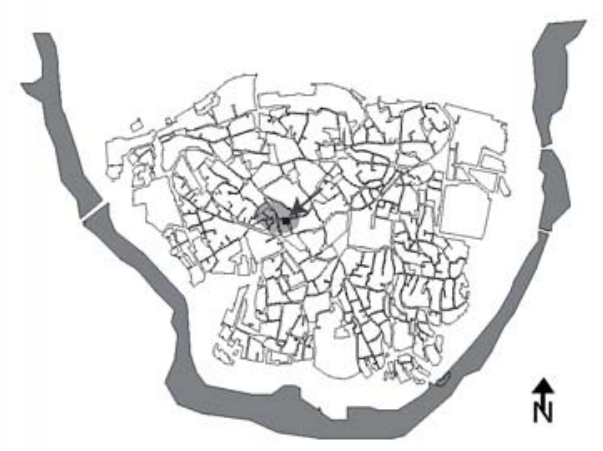

Figura 1. El barrio de Caleros en la ciudad de Toledo.

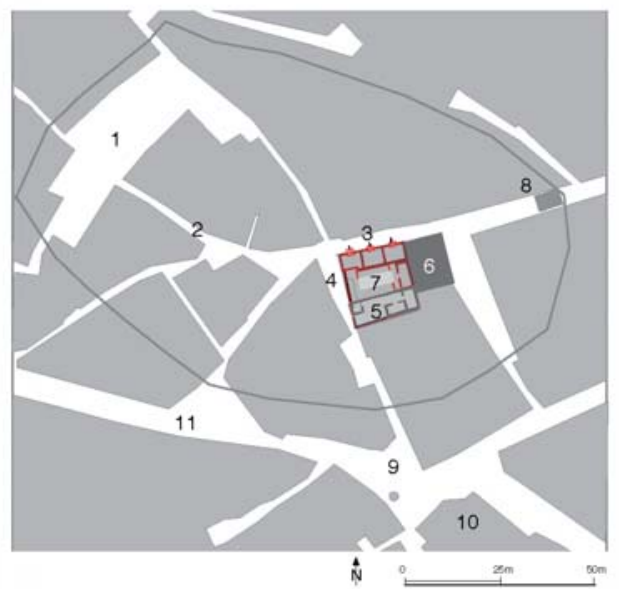

Figura 2. El barrio de Caleros hacia 1410.
1. Plaza, hoy de Valdecaleros
2. Calle de Algibillo
3. Calle de Caleros (hoy calle de Alfonso XII)
4. Calle hacia la iglesia de San Salvador
5. Calle tributaria al hospital de la Misericordia
6. Sinagoga de Calero
7. Casa tributaria a Santo Domingo el Real
8. Cobertizo del arcediano de Niebla
9. Plaza del Pozo de San Salvador
10. Iglesia de San Salvador
11. Calle a Santo Tomé
Límites aproximativos. 

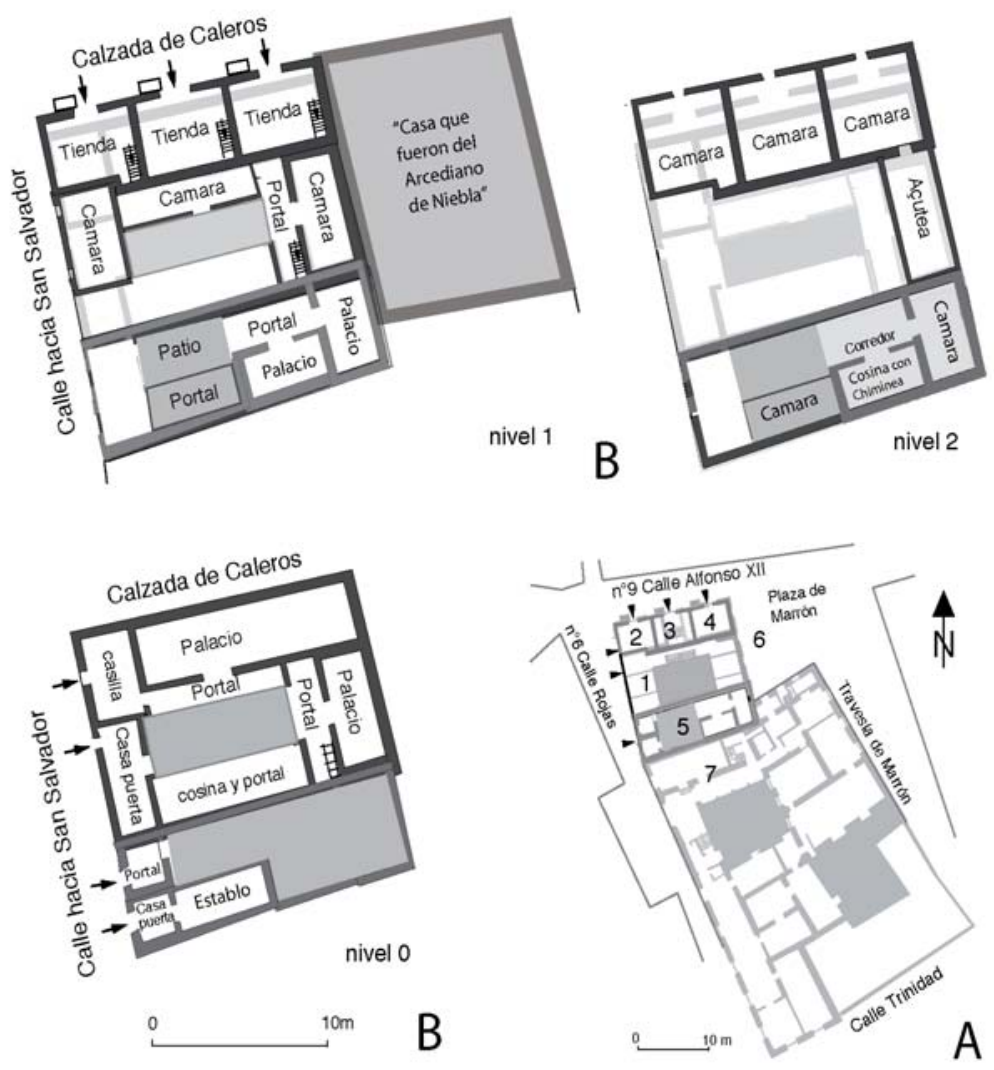

Figura 3. La casa y las tres tiendas de Teresa Téllez y sus aledaños.

A. Localización en la manzana actual: 1. SD [= Santo Domingo]-74, casa con patio; 2. SD-75, tienda; 3. SD-76, tienda; 4. SD-77, tienda; 5. HM-90a, casa del hospital de la Misericordia.

B. Planimetría y restitución del conjunto en 1460 . 


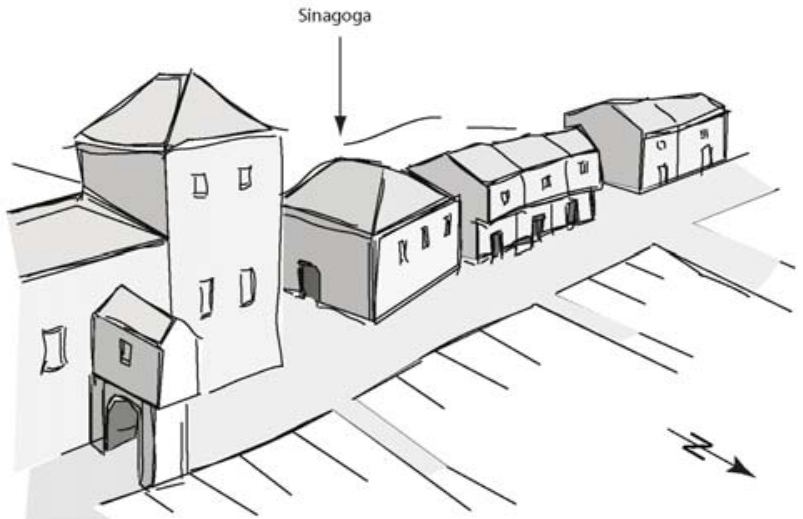

Figura 4. Restitución de la calle de Caleros a final del siglo XIV: cobertizo, sinagoga y tiendas.

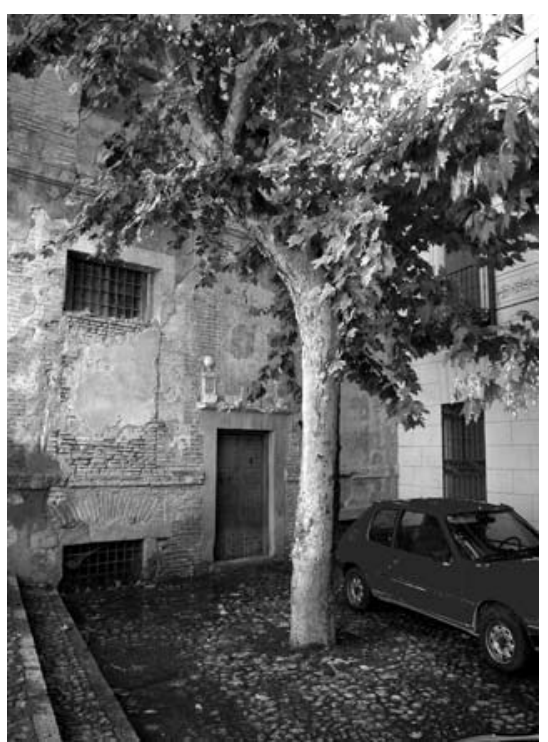

Figura 5. El sitio de la antigua sinagoga de Caleros, 2002. 